\title{
CONCERNING CONTINUOUS COLLECTIONS OF CONTINUOUS CURVES
}

\author{
MARY-ELIZABETH HAMSTROM
}

E. E. Moise has shown ${ }^{1}$ that no compact irreducible continuum is filled up by a continuous collection of mutually exclusive arcs which is an arc with respect to its elements. The object of this paper is to present some extensions of this result. In the lemma and in Theorem 1, $M$ will denote a compact metric continuum.

LеммA. If the domain $D$ intersects a nondegenerate element $z$ of a continuous collection $G$ of mutually exclusive continuous curves which fills up a compact metric continuum $M$ and is an arc with respect to its elements but $\bar{D}$ does not contain $z$, then for each element $y$ of $G$ distinct from $z$ there is a connected subset of $\bar{D} \cdot M$ which intersects two elements of $G$ distinct from $y$ on the interval zy of elements of $G$.

Proof. Suppose the lemma is false. There exists an element $a$ of $G$ such that if $b$ and $c$ are elements of the interval $z a$ of $G$ distinct from $a$, then no connected subset of $M \cdot \bar{D}$ intersects both $b$ and $c$. Let $P$ be a point of $z \cdot D$ and let $R$ and $D^{\prime}$ be domains containing $P$ such that $\bar{D}^{\prime}$ is a subset of $R$ and $\bar{R}$ is a subset of $D$. Since $G$ is continuous, there exists, between $z$ and $a$, an element $x$ of $G$ such that $x$, and every element of $G$ between $x$ and $z$, intersects both $D^{\prime}$ and $S-\bar{D}$. Let $M^{\prime}$ denote the sum of $x, z$, and all elements of $G$ between them. Since no connected subset of $M^{\prime} \cdot \bar{D}$ intersects both $x$ and $z, M^{\prime} \cdot \bar{D}$ is the sum of two mutually separated point sets $K_{1}$ and $L_{1}$ which contain $M^{\prime} \cdot z$ and $M^{\prime} \cdot x$ respectively.

Let $y_{1}$ denote the first element of $G$ between $z$ and $x$ that intersects $L_{1} \cdot \bar{D}^{\prime}$. The element $y_{1}$ does not intersect $L_{1} \cdot D^{\prime}$, for, otherwise, the fact that $G$ is continuous would imply the existence of an element of $G$ between $z$ and $y_{1}$ which intersects $L_{1} \cdot D^{\prime}$. However, $y_{1}$ does intersect $D^{\prime}$; therefore it intersects $D^{\prime} \cdot K_{1}$. But it also intersects $L_{1} \cdot R$. Hence there is an element $x_{1}$ of $G$ between $z$ and $y_{1}$ such that $x_{1}$, and each element of $G$ between it and $y_{1}$, intersects $K_{1} \cdot D^{\prime}$ and $L_{1} \cdot R$, because both $K_{1} \cdot D^{\prime}$ and $L_{1} \cdot R$ are open subsets of $M$.

No connected subset of $K_{1}$ intersects $x_{1}$ or some element of $G$ preceding $x_{1}$ and also intersects $y_{1}$ or some element of $G$ following $y_{1}$ in the order from $z$ to $x$. Therefore $K_{1}$ is the sum of two mutually sepa-

Presented to the Society, December 28, 1951; received by the editors June 9, 1952.

1 E. E. Moise, A Theorem on Monotone Interior Transformations, Bull. Amer. Math. Soc. vol. 55 (1949) pp. 810-811. 
rated point sets $K_{2}$ and $L_{2}$ such that $K_{2}$ contains the common part of $K_{1}$ and the sum of all the elements of the interval $z x_{1}$ of $G$ and $L_{2}$ contains the common part of $K_{1}$ and the sum of all the elements of the interval $y_{1} x$.

Let $y_{2}$ denote the first element of $G$ between $z$ and $x$ that intersects $L_{2} \cdot \bar{D}^{\prime}$. The element $y_{2}$ precedes $y_{1}$ in the order from $z$ to $x$ and therefore does not intersect $L_{1} \cdot D^{\prime}$. Since $G$ is continuous $y_{2}$ does not intersect $L_{2} \cdot D^{\prime}$. Consequently it intersects $K_{2} \cdot D^{\prime}$. Since it intersects $L_{2}$ it follows $x_{1}$ in the order from $z$ to $x$ and therefore intersects $L_{1} \cdot R$.

The elements chosen are in the order $z, x_{1}, y_{2}, y_{1}, x$. Since $y_{2}$ intersects $L_{2} \cdot R, L_{1} \cdot R$, and $K_{2} \cdot D^{\prime}$, there is, between $x_{1}$ and $y_{2}$, an element $x_{2}$ of $G$ such that every element of the interval $x_{2} y_{2}$ of $G$ intersects $L_{2} \cdot R, L_{1} \cdot R$, and $K_{2} \cdot D^{\prime}$. No connected subset of $K_{2}$ intersects $x_{2}$ or some element of $G$ preceding it and also intersects $y_{2}$ or some element following it. Therefore $K_{2}$ is the sum of two mutually separated point sets $K_{3}$ and $L_{3}$ such that $K_{3}$ contains the common part of $K_{2}$ and the sum of all the elements of the interval $z x_{2}$ and $L_{3}$ contains the common part of $K_{2}$ and the sum of all the elements of $y_{2} x$.

Let $y_{3}$ be the first element of $G$ between $z$ and $x$ that intersects $L_{3} \cdot \bar{D}^{\prime}$. The element $y_{3}$ precedes $y_{2}$ and follows $x_{2}$. Therefore, as may be seen with the aid of arguments similar to the preceding, $y_{3}$ intersects $K_{3} \cdot D^{\prime}, L_{3} \cdot R, L_{2} \cdot R$, and $L_{1} \cdot R$. The elements chosen are in the order $z, x_{1}, x_{2}, y_{3}, y_{2}, y_{1}, x$.

Continuing in this manner we obtain sequences $L_{1}, L_{2}, L_{3}, \cdots$, $K_{1}, K_{2}, K_{3}, \cdots$, and $y_{1}, y_{2}, y_{3}, \cdots$ such that for each positive integer $n$, (1) $K_{n}$ is the sum of $K_{n+1}$ and $L_{n+1}$ where $K_{n+1}$ and $L_{n+1}$ are mutually separated, (2) $K_{n}+L_{n}$ is a subset of $D$, (3) $y_{n+1}$ precedes $y_{n}$ and follows $x_{1}$ in the order from $z$ to $x$, and (4) if $n$ is not less than $i, y_{n}$ intersects $L_{i} \cdot R$. The sequence $y_{1}, y_{2}, y_{3}, \cdots$ has a sequential limiting set, $y$, which is an element of $G$ between $z$ and $x$. Since for each $n$ infinitely many elements of the sequence $y_{1}, y_{2}, y_{3}, \cdots$ intersect $L_{n} \cdot \bar{R}, y$ intersects $L_{n} \cdot \bar{R}$.

For each positive integer $i$ let $Q_{i}$ denote a point of the common part of $y$ and $L_{i} \cdot \bar{R}$ and let $Q$ be a limit point of the sequence $Q_{1}, Q_{2}, Q_{3}, \cdots$. Since, for each $n, K_{n}$ and $L_{n}$ are mutually separated and $L_{i}$ is a subset of $K_{n}$ if $i$ is greater than $n, Q$ is not a point of $L_{n}$. Therefore it is a point of $K_{n}$ for each $n$. Since $y$ is a continuous curve there exists a domain $h$ containing $Q$ such that $\bar{h}$ is a subset of $D$ and $y \cdot h$ is connected. The point set $y \cdot h$ is a domain with respect to $y$. Therefore there exists a positive integer $j$ such that $Q_{j}$ is a point of $y \cdot h$. However, $Q_{j}$ is a point of $L_{j}$ and $Q$ is a point of $K_{j}$, so $y \cdot h$ is a connected subset of $M^{\prime}$ intersecting $K_{j}$ and $L_{j}$. This is impossible 
since $M^{\prime}$ is the sum of the mutually separated point sets $K_{j}, L_{j}$, $L_{j-1}, \cdots$ and $L_{1}$. This involves a contradiction. Hence the lemma is proved.

Theorem 1. If a continuous collection $G$ of mutually exclusive continuous curves, not all degenerate, is an arc with respect to its elements, then the sum of all the elements of $G$ is not irreducible.

Proof. Let $a$ and $b$ denote the end elements, and let $M$ denote the sum of all the elements, of $G$, whence $M$ is a compact metric continuum. If $P$ and $Q$ are points of the same element of $G, M$ is not irreducible between $P$ and $Q$. If $P$ is a point of the element $x$ and $Q$ of the element $y$ and either $x$ or $y$ is not an end element of $G$, the sum of $x, y$ and all elements of $G$ between them is a proper subcontinuum of $M$ and therefore $M$ is not irreducible between $P$ and $Q$.

Suppose $P$ is a point of $a$ and $Q$ is a point of $b$. Let $z$ denote a nondegenerate element of $G$ distinct from $a$ and $b$ and let $D$ be a domain intersecting $z$ but such that $z$ is not a subset of $\bar{D}$. Let $y$ be an element of $G$ between $z$ and $b$ such that every element other than $z$ of the interval $z y$ intersects $D$ and $S-\bar{D}$. It follows from the lemma, however, that there are elements $x$ and $x^{\prime}$ of $G$ between $z$ and $y$ such that $x^{\prime}$ is between $x$ and $y$ and that there is a continuum $K$ such that $K$ is a subset of $\bar{D} \cdot M$ and intersects both $x$ and $x^{\prime}$. Let $L$ be the sum of $K, x$, and all elements of $G$ preceding $x$ in the order from $a$ to $b$ together with $x^{\prime}$ and all elements following it in the order from $a$ to $b$. The point set $L$ is a proper subcontinuum of $M$ and contains $P$ and $Q$. Therefore $M$ is not irreducible.

THEOREм 2. If the continuous collection $G$ of mutually exclusive arcs fills up the compact continuum $M$ in the plane and is an arc with respect to its elements, then $M$ is a domain plus its boundary.

Proof. Suppose the domain $D$ contains the point $P$ of the element $z$ of $G$. Let $J$ denote a simple closed curve whose interior $I$ contains $P$ such that $J+I$ lies in $D$ and does not contain $z$. Let $U$ be a domain containing $P$ such that $\bar{U}$ is a subset of $I$. Let $y$ be an element of $G$ such that every element of the interval $y z$ of $G$ contains a point of $U$ and a point not in $J+I$. There are elements $x$ and $x^{\prime}$ of $G$ between $z$ and $y$ and a continuum $K$ such that $K$ is a subset of $M \cdot \bar{U}$ intersecting $x$ and $x^{\prime}$.

Let $Q$ be a point of $x \cdot I$ not in $\bar{U}$. Let $V$ be a domain containing $Q$ such that $\bar{V}$ is a subset of $I-\bar{U}$. Let $y^{\prime}$ be an element of $G$ between $x$ and $x^{\prime}$ such that every element of the interval $x y^{\prime}$ of $G$ except $x$ contains a point of $V$ and a point not in $J+I$. There are elements $w$ 
and $w^{\prime}$ of $G$ between $x$ and $y^{\prime}$ and a continuum $K^{\prime}$ such that $K^{\prime}$ is a subset of $M \cdot \bar{V}$ intersecting both $w$ and $w^{\prime}$.

Since they are between $x$ and $x^{\prime}, w$ and $w^{\prime}$ intersect $K$. Since $\bar{U}$ and $\bar{V}$ are mutually separated, the common part of $K+w$ and $K^{\prime}+w^{\prime}$ is not connected and $K+w+K^{\prime}+w^{\prime}$ is a continuum, $L$, which separates the plane. The point set $S-L$ is the sum of two mutually separated point sets $R$ and $T$ such that $T$ is unbounded and connected. However, since no element of $G$ separates the plane and $G$ is an arc with respect to its elements, $S-M$ is connected and is consequently a subset of $T$. Hence $R$ is a subset of $M$. Let $W$ be a component of $R$. The point set $W$ is a complementary domain of $L$ and its boundary, $B$, separates $S$. The continuum $B$ is a subset of $L$ and, since no subset of $w+w^{\prime}$ separates $S, B$ contains a point of $K+K^{\prime}$ not in $w+w^{\prime}$. There is, consequently, a point of $W$ in $D \cdot M$ and $D \cdot M$ contains a domain.

We have shown that every domain which intersects $M$ contains a domain which is a subset of $M$. This is sufficient to show that $M$ is a domain plus its boundary.

Theorem 3. Under the hypotheses of Theorem 2, if $M$ is a continuous curve it is a simple closed curve plus its interior.

Proof. Let $B$ denote the boundary of $M$. Since $S-M$ is connected, $B$ is connected. It has no cut point, for if $P$ is a point of $B$ and $B-P$ is not connected, $M-P$ is the sum of two mutually separated point sets $K$ and $L$. Let $z$ denote the element of $G$ containing $P$. Every other element of $G$ is a subset either of $L$ or of $K$. From this and the fact that $G$ is a continuous collection it follows that $z$ does not intersect $K$ or $L$. This contradicts the fact that $z$ is an arc. Hence $B$ has no cut point. But it is a continuous curve and is the boundary of a domain. It follows that it is a simple closed curve.

The University of Texas 